\title{
Limitation of current probe design for oligo-cross-FISH, exemplified by chromosome evolution studies in duckweeds
}

\author{
Phuong T. N. Hoang ${ }^{1,2}$ (D) Jean-Marie Rouillard ${ }^{3,4}$ (D) Jiří Macas $^{5}$ (D) $\cdot$ Ivona Kubalová ${ }^{1} \cdot$ Veit Schubert $^{1}$ (D) \\ Ingo Schubert ${ }^{1}$ (1)
}

Received: 18 September 2020 / Revised: 23 December 2020 / Accepted: 28 December 2020 / Published online: 14 January 2021

(C) The Author(s) 2021

\begin{abstract}
Duckweeds represent a small, free-floating aquatic family (Lemnaceae) of the monocot order Alismatales with the fastest growth rate among flowering plants. They comprise five genera (Spirodela, Landoltia, Lemna, Wolffiella, and Wolffia) varying in genome size and chromosome number. Spirodela polyrhiza had the first sequenced duckweed genome. Cytogenetic maps are available for both species of the genus Spirodela (S. polyrhiza and S. intermedia). However, elucidation of chromosome homeology and evolutionary chromosome rearrangements by cross-FISH using Spirodela BAC probes to species of other duckweed genera has not been successful so far. We investigated the potential of chromosome-specific oligo-FISH probes to address these topics. We designed oligo-FISH probes specific for one S. intermedia and one S. polyrhiza chromosome (Fig. 1a). Our results show that these oligo-probes cross-hybridize with the homeologous regions of the other congeneric species, but are not suitable to uncover chromosomal homeology across duckweeds genera. This is most likely due to too low sequence similarity between the investigated genera and/or too low probe density on the target genomes. Finally, we suggest genus-specific design of oligo-probes to elucidate chromosome evolution across duckweed genera.
\end{abstract}

Keywords Duckweeds $\cdot$ Spirodela $\cdot$ Landoltia $\cdot$ Lemna $\cdot$ Karyotype evolution $\cdot$ Dual-color oligo-FISH $\cdot$ Microsatellites $\cdot$ Structured illumination microscopy $\cdot$ Wolffia $\cdot$ Wolffiella

\section{Introduction}

Duckweeds comprise together 36 species within five genera: Spirodela (2), Landoltia (1), Lemna (12), Wolffiella (10), and Wolffia (11). They represent an emerging aquatic crop for feed, food, and biofuel generation, as well as for waste water remediation, due to their fast growth rate, optimal protein

Ingo Schubert

schubert@ipk-gatersleben.de

1 Leibniz Institute of Plant Genetics and Crop Plant Research (IPK), Gatersleben, 06466 Stadt Seeland, Germany

2 Present address: Biology Department, Dalat University, District 8, Dalat City, Lamdong Province, Vietnam

3 Arbor Biosciences, Ann Arbor, MI 48 102, USA

4 Chemical Engineering Department, University of Michigan, Ann Arbor, MI, USA

5 Biology Centre, Czech Academy of Sciences, Institute of Plant Molecular Biology, CZ 37005 České Budějovice, Czech Republic profile, and their ability to accumulate minerals and heavy metals (Tippery et al. 2015; Zhao et al. 2015; Appenroth et al. 2017; de Beukelaar et al. 2019; Kaur et al. 2019; Kreider et al. 2019; Yaskolka Meir et al. 2019; Bog et al. 2020; Nahar and Sunny 2020). From the ancient genus Spirodela towards the most derived genus Wolffia, organismic complexity (many roots versus no roots) and size $(1.5 \mathrm{~cm}$ to less than $1 \mathrm{~mm}$ in diameter), genome size (from $160 \mathrm{Mbp}$ to $2.2 \mathrm{Gbp}$ ), and chromosome number vary considerably within and between genera (Landolt 1986; Wang et al. 2011; Hoang et al. 2019). Because of these features, duckweeds are an interesting subject for physiological, developmental and evolutionary studies.

The Greater Duckweed, S. polyrhiza, was the first duckweed for which a high-quality genome map was generated by integrating results of different approaches such as cytogenomics, optical mapping (BioNano technique), Hi-C conformation study, 454, Illumina, and Oxford Nanopore sequencing platforms (Wang et al. 2014; Cao et al. 2016; Michael et al. 2017; Hoang et al. 2018; Harkess et al. 2020). So far, cytogenetic maps, based on chromosomal localization of $\sim 100$ 
anchored BACs, revealed no structural rearrangements between the genomes of seven investigated S. polyrhiza accessions of different geographic origin, suggesting a considerable homogeneity between these asexually propagating clones.

$S$. polyrhiza $(2 n=40)$ and $S$. intermedia $(2 n=36)$, both with a genome size of $160 \mathrm{Mbp} / 1 \mathrm{C}$, are the only two species of the genus Spirodela. Previous cross-FISH with 93 anchored S. polyrhiza-specific BAC probes discovered chromosome homeology and several rearrangements between S. polyrhiza and S. intermedia karyotypes (Hoang and Schubert 2017). These data suggest a considerable infrageneric genome diversity among duckweeds. Cross-FISH with S. polyrhiza-specific BAC probes to species of other duckweed genera (Landoltia punctata, Lemna aequinoctialis, Wolffiella hyalina, Wolffia arrhiza) yielded only weak and dispersed, but no reliable chromosome-specific signals, even under highly stringent conditions (Hoang 2019).

Cross-FISH with BACs (Lysak et al. 2006; Mandakova and Lysak 2008; Ma et al. 2010) or oligo-probes (AurichCosta et al. 2007; Han et al. 2015; Braz et al. 2018; Simonikova et al. 2019; Liu et al. 2020; Xin et al. 2020) were shown to be powerful for comparative chromosome studies, by labeling homeologous chromosomes or chromosome regions of related taxa.

Here, we attempted to apply chromosome-specific oligoprobes for cross-FISH on duckweeds. Oligo-probes were designed for S. intermedia chromosome $\mathrm{ChrSi09}$ and S. polyrhiza chromosome ChrSp19 (Fig. 1a), based on chromosome-scale sequence assemblies for both species. The former chromosome corresponds to $S$. polyrhiza ChrSp08 and ChrSp18 and the latter one to $S$. intermedia chromosome ChrSil7 (Hoang and Schubert 2017; Hoang et al. 2020). While these oligo-probes hybridized nicely on the original chromosomes and labeled the homeologs of the sister species as well, none or only very weak and dispersed, but no chromosome-specific signals appeared after crossFISH on mitotic chromosomes of duckweed species of other genera. Blocking of some microsatellite sequences within the oligo-probes, which may cross-hybridize to dispersed repeats of La. punctata, did not improve signal specificity. Thus, oligo-FISH across duckweed genera remains a challenge when the density of oligos which find homologous sequences within a distinct region of the target genome falls below a threshold required for a reliable FISH signal and may require a different probe design strategy. Therefore, we suggest to design synteny-based oligo-probes for each genus and to filter out microsatellite-containing oligos for studying chromosome evolution across duckweed genera.

\section{Material and methods}

\section{Plant material}

S. polyrhiza (clone 7498), S. intermedia (8410 and 7747), La. punctata (7260), Le. aequinoctialis (clone 2018), Wa. hyalina

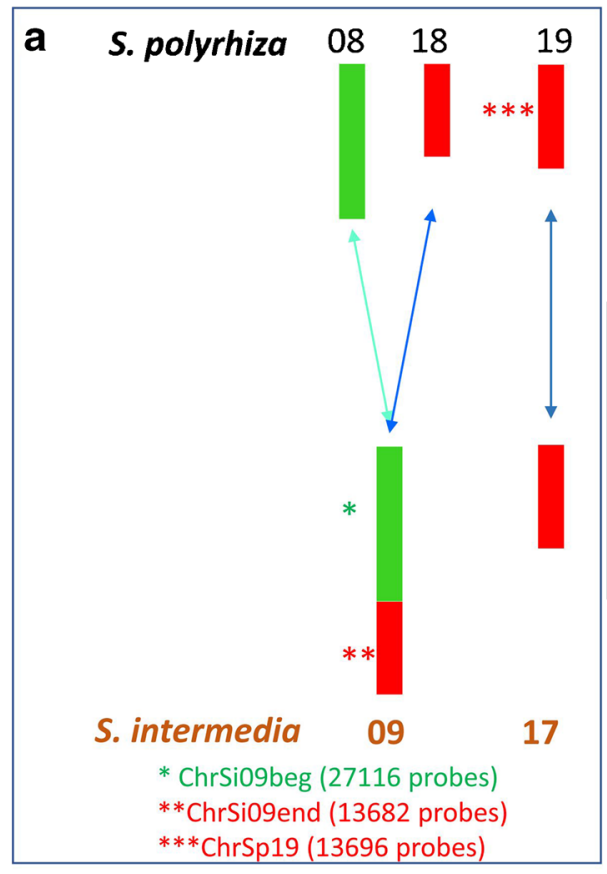

Fig. 1 Oligo-FISH confirmed chromosome fusion in $S$. intermedia. a Scheme of homeologous chromosomes of $S$. polyrhiza and $S$. intermediaused for oligo-probe design; b oligo-probes labeled three different chromosome pairs in S. polyrhiza; $\mathbf{c}, \mathbf{d}$ the co-localization of
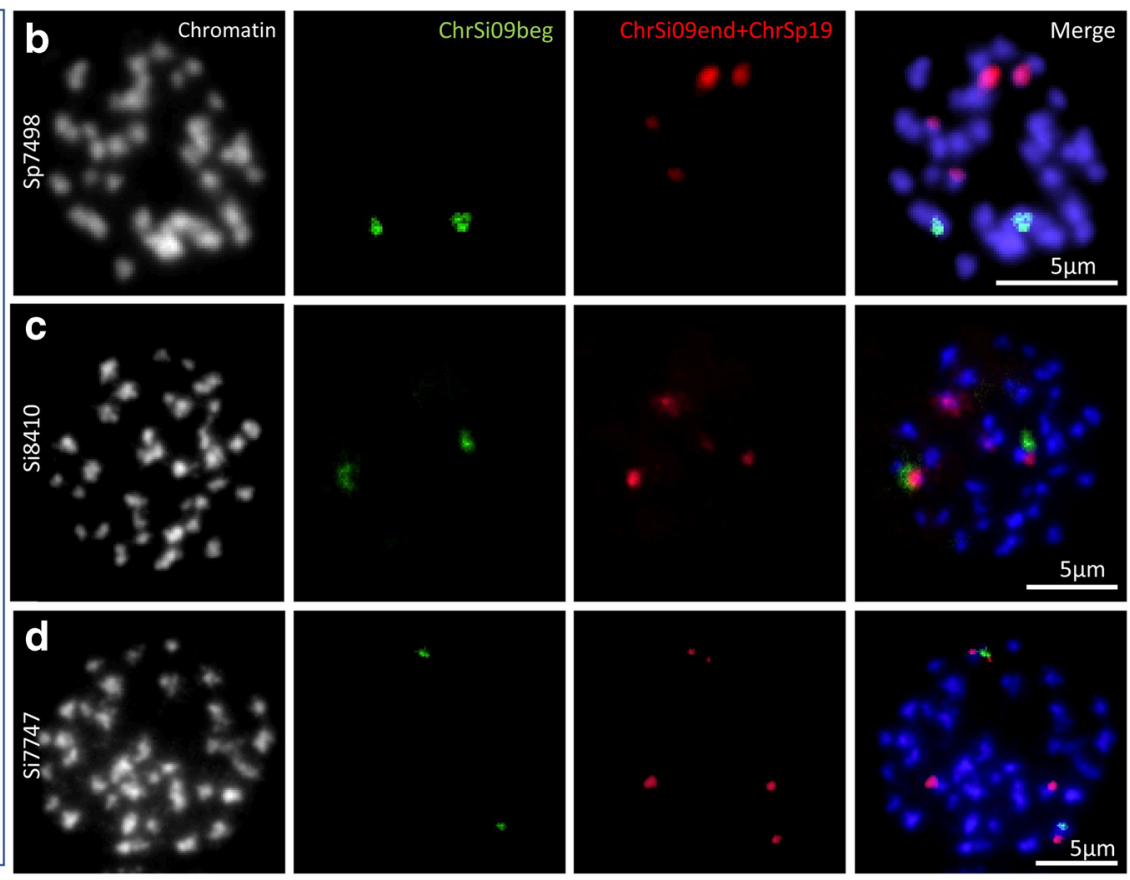

green (ChrSi09beg) and red (ChrSi09end) signals which label ChrSp08 and $\mathrm{ChSp} 18$, respectively, confirmed their combination into $\mathrm{ChrSi} 09$ of S. intermedia (clones 8410 and 7747) 
(8640), and Wo. australiana (7540) were obtained from Elias Landolt's collection via BIOLEX (Pittsboro, NC, USA) via K.-J. Appenroth, Friedrich Schiller University, Jena, Germany, and from Rutgers Duckweed Stock Cooperative (New Jersey, USA). Liquid nutrient medium (Appenroth et al. 1996) was used to grow fronds under $16 \mathrm{~h}$ white light of $100 \mu \mathrm{mol} \mathrm{m}{ }^{-2} \mathrm{~s}^{-1}$ at $24{ }^{\circ} \mathrm{C}$.

\section{Probe design}

Oligonucleotide probes were designed using Abor Biosciences' proprietary software. Briefly, target sequences are cut into 43-47 nucleotides-long overlapping probe candidate sequences that are compared to the rest of the genome sequence to check for potential cross-hybridization based on a predicted Tm of hybridization. Non-overlapping candidates with no cross-hybridization were blasted against the homeologous Spirodela chromosome. Candidates with a single hit on the homeologous chromosome with an $E$-value less than $1 \mathrm{E}-05$ were selected for the final set. This $E$-value cutoff return probes with at least $75 \%$ sequence similarity over the entire probe length or a higher similarity over a shorter section of the probe sequence. Probe hybridization to other genera genomes were predicted using the same blast $E$-value cutoff. Probe/target melting temperatures were predicted using the nearest-neighbor model with a $330 \mathrm{mM}$ sodium concentration ( $2 \times \mathrm{SSC}$ used in post-hybridization washes).

\section{Genome sequencing and repetitive DNA analysis}

Whole genome shotgun sequencing of La. punctata clone 7260 was performed by Admera Health, LLC (South Plainfield, NJ, USA) using a KAPA DNA Library kit (Roche) and Illumina platform generating $2 \times 151 \mathrm{nt}$ pairedend reads. The reads were deposited to the European Nucleotide Archive (https://www.ebi.ac.uk/ena) under accession number ERR4463159.

For repeat analysis, the reads were trimmed to $142 \mathrm{nt}$ and quality-filtered. A total of 1.8 million randomly sampled paired reads was then used for repeat identification by similarity-based clustering implemented in the RepeatExplorer pipeline (Novák et al. 2013). The pipeline was run with default parameters except for the similarity search options where masking of low complexity regions was disabled. The resulting clusters containing at least $0.005 \%$ of the input reads and thus representing highly or moderately repeated elements were annotated and quantified. Additionally, the TAREAN pipeline (Novák et al. 2017) was employed to specifically search for tandem repeats, using 1.4 million input reads. FISH probes for the satellite LDP SAT were designed based on the satellite consensus monomer sequence reported by TAREAN. The (partially overlapping) probe sequences were 5'-GCG AAA CTT GCC CGA AAT
AGC AAA ATC GCC GTT TCT GGC CTA T-3' (LDP SAT-H1) and 5'-CGA AAT AGC AAA ATC GCC GTT TCT GGC CTA TCC GGG GGC CTT TTC GG-3' (LDP_SAT-H2).

\section{Mitotic chromosome preparation}

Spreading of mitotic chromosomes was carried out according to Hoang and Schubert (2017). In brief, healthy fronds were fixed in fresh 3:1 absolute ethanol:acetic acid for at least $24 \mathrm{~h}$ after treatment with $2 \mathrm{mM} 8$-hydroxyquinoline at $37{ }^{\circ} \mathrm{C}$ for $3.5 \mathrm{~h}$. Before and after softening in $2 \mathrm{~mL}$ PC enzyme mixture [ $1 \%$ pectinase and $1 \%$ cellulase in Na-citrate buffer, $\mathrm{pH} 4.6$ ] for $90 \mathrm{~min}$ at $37^{\circ} \mathrm{C}$, samples were washed twice in $10 \mathrm{mM} \mathrm{Na}$ citrate buffer, $\mathrm{pH} 4.6$, for 10 min each. After softening, samples were transferred on slides and all tissue except the meristem region was removed. Meristems were macerated and squashed in $45 \%$ acetic acid. Slides were frozen in liquid nitrogen for $5 \mathrm{~min}$. After carefully removing of coverslips with a razor blade, slides were treated with pepsin $[50 \mu \mathrm{g} /$ $\mathrm{mL}$ in $0.01 \mathrm{~N} \mathrm{HCl}$ ] for $5 \mathrm{~min}$ at $37{ }^{\circ} \mathrm{C}$, post-fixed in $4 \%$ formaldehyde in $2 \times \mathrm{SSC}$ [300 mM Na-citrate, $30 \mathrm{mM} \mathrm{NaCl}$, $\mathrm{pH} 7.0$ ] for $10 \mathrm{~min}$, rinsed twice in $2 \times \mathrm{SSC}, 5 \mathrm{~min}$ each, dehydrated in an ethanol series (70,90, and 96\%, 2 min each), and air-dried.

\section{Probe preparation}

The Arabidopsis thaliana type telomere probe was generated by PCR using TTTAGGG-tetramers without template DNA according to (Ijdo et al. 1991). The product was labeled with Alexa Fluor 488-5-dUTP by PCR (100 ng telomere PCR product in $25 \mu \mathrm{L}$ reaction mixture), ethanol precipitated (Mandakova and Lysak 2008), dissolved in $250 \mu \mathrm{L}$ DS20 buffer $[50 \%(\mathrm{v} / \mathrm{v})$ formamide, $20 \%(\mathrm{w} / \mathrm{v})$ dextran sulfate in $2 \times \mathrm{SSC}, \mathrm{pH} 7]$ at $37{ }^{\circ} \mathrm{C}$ for at least $1 \mathrm{~h}$, and stored at -20 ${ }^{\circ} \mathrm{C}$ (Hoang and Schubert 2017).

MyTags ${ }^{\circledR}$ ChrSi09beg green-labeled probes (ATTO-488) and ChrSi09end/ChrSp19 red-labeled probes (ATTO-594) were obtained from Arbor Biosciences (Ann Arbor, USA). Microsatellite probes were obtained from Eurofins. Lyophilized probes were dissolved in TE buffer [10 mM Tris- $\mathrm{HCl}, 1 \mathrm{mM} \mathrm{Na} \mathrm{m}_{2}$-EDTA] to a final concentration of 10 $\mathrm{pmol} / \mu \mathrm{L}$, aliquoted, and frozen.

\section{Oligo-FISH}

After adding $100 \mu \mathrm{L}$ of $70 \%$ formamide in $2 \times$ SSC on chromosome spreads, they were covered with parafilm, and denatured on a heating plate for $2.5 \mathrm{~min}$ at $70^{\circ} \mathrm{C}$. After removing the parafilm, slides were dipped in pre-cooled ethanol series $(70,90$, and $96 \%)$ for 5 min each on a shaker and air-dried. 
Twenty microliters of hybridization mixture (50\% formamide, $2 \times \mathrm{SSC}, 20 \%$ dextran sulfate with $4 \mu \mathrm{L}$ of freshly prepared ATTO 488 and/or $2 \mu \mathrm{L}$ of ATTO 594-labeled probes in DS20 buffer) was used for each slide. The stringency for hybridization was 84.6 and for post-hybridization washing 89.6. In the case of the blocking experiment, all probes (ChrSi09beg and ChrSi09end, ChrSp19 with $(\mathrm{GA})_{15}$ and (GAA) $)_{10}$ ) were pooled together, evaporated under vacuum, and dissolved in 1 $\mu \mathrm{L}$ of $\mathrm{ddH}_{2} \mathrm{O}$ and $15 \mu \mathrm{L}$ of DS20 buffer. The entire volume was applied onto the slide. Slides were carefully covered by a coverslip to prevent air bubbles inside, and sealed with a line of rubber cement. Chromosome preparations were denatured together with the probes on a heating plate at $70{ }^{\circ} \mathrm{C}$ for $3 \mathrm{~min}$ and then incubated in a moist chamber at $37^{\circ} \mathrm{C}$ for at least 36 h. Post-hybridization washing was carried out as follows: slides were briefly washed in $2 \times \mathrm{SSC}$ at room temperature to remove the coverslip, then washed under shaking condition at $42{ }^{\circ} \mathrm{C}$ for $20 \mathrm{~min}$ for $5 \mathrm{~min}$ in $2 \times \mathrm{SSC}$ at room temperature, dehydrated in an ethanol series (70, 90, and 96\%, 2 min each), air-dried in the dark, and counterstained with $10 \mu \mathrm{L}$ DAPI (2 $\mu \mathrm{g} / \mathrm{mL}$ in Vectashield).

\section{Microscopy and image processing}

Widefield fluorescence microscopy for signal detection followed Cao et al. (2016). The images were processed (brightness and contrast adjustment only) and merged using Adobe Photoshop software ver.12 × 32 (Adobe Systems).

To analyze the ultrastructure and spatial arrangement of signals and chromatin at a lateral resolution of $\sim 120 \mathrm{~nm}$ (super-resolution, achieved with a $488 \mathrm{~nm}$ laser), 3D-structured illumination microscopy (3D-SIM) was applied using a PlanApochromat $63 \times / 1.4$ oil objective of an Elyra PS.1 microscope system and the software ZENblack (Carl Zeiss $\mathrm{GmbH})$. Image stacks were captured separately for each fluorochrome using the 405, 488, and $561 \mathrm{~nm}$ laser lines for excitation and appropriate emission filters (Weisshart et al. 2016). Maximum intensity projections of whole cells were calculated via the ZEN software. Zoom-in sections were presented as single slices to indicate the subnuclear chromatin structures at the super-resolution level.

\section{Results}

\section{Probe design for S. intermedia and S. polyrhiza}

A set of 27,116 probes was designed to cover the first $7.79 \mathrm{Mb}$ of $S$. intermedia ChrSi09 (Si09:1-7790000, referred to as ChrSi09beg) and to maintain cross-hybridization capabilities with S. polyrhiza ChrSp08. Another set of 13,682 probes was designed against the rest of ChrSi09 (Si09:779000012648911, referred to as ChrSi09end), maintaining cross- hybridization capabilities with $S$. polyrhiza ChrSp18. Finally, a set of 13,696 probes was designed to cover the entire S. polyrhiza ChrSp19 (ChrSp19:1-3959484) with the ability to hybridize to $S$. intermedia ChrSi17.

As a way to compare probe density along chromosomes and account for potential regions within which probes cannot hybridize to otherwise homeologous chromosomes in species from other genera (for instance due to larger insertions), we defined the Density 100 index (D100) as the probe density of a moving window of a contiguous set of 100 probes expressed in probes/kb of DNA covered by these 100 probes. Each probe set can be described as a collection of D100. Median D100 is used to compare probe set's potential hybridization to corresponding target chromosomes (Table 1). The three probe sets have D100 medians of 4.53, 3.69, and 5.01 probes per kb on their chromosome of origin, ChrSi09beg, ChrSi09end, and ChrSp19, respectively. The D100 medians for hybridization to reciprocal homeologous Spirodela chromosomes are very similar (4.34, 3.47, and 5.24 for ChrSp08, ChrSp18, and ChrSi17, respectively).

We also computed the theoretical $T m$ value of each probe hybridized to its target in several duckweed genomes (Fig. S5). The medians of the Tm distributions for hybridization of the three probe sets to their sequences of origin are around 76-77 ${ }^{\circ} \mathrm{C}$, Fig. S5). These median $\mathrm{Tm}$ values drop by about 10 ${ }^{\circ} \mathrm{C}$ when computed for the entire probe sets hybridizing to the homeologous Spirodela chromosomes due to sequence divergences between the two species. For Landoltia, Lemna, and Wolffia, the probe number was strongly reduced to those probes that are expected to hybridize stably. Therefore, the $T m$ values drop less than in the intra-genus comparison.

Probes designed from $\mathrm{ChrSi09end} \mathrm{and} \mathrm{ChrSp19} \mathrm{were} \mathrm{syn-}$ thesized as a single set and labeled separately from probe designed from ChrSi09beg, enabling two-color hybridizations.

\section{Oligo-cross-FISH confirmed "chromosome fusion" in S. intermedia}

Using 93 BACs anchored in the $S$. polyrhiza genome, and a suitable BAC pooling system, a cytogenetic map for S. intermedia clone 8410 has been established (Hoang and Schubert 2017). At first, we designed oligo-probes to confirm the evolutionary "fusion" of ChrSp08 and ChrSp18 into ChrSi09 (or the split of ChrSi09 into ChrSp08 and ChrSp18), as previously found by cross-FISH with six ChrSp08 BACs (013I04, 006P24, 032L08, 034K03, 004E01, and 006L17) and three ChrSp18 BACs (026D06, 037B13, and 029K19) (Hoang and Schubert 2017).

In order to test the specificity of the synthetic oligo-probe sets, they were hybridized to chromosome spreads of S. polyrhiza (clone 7498) and S. intermedia (clones 8410 and 7747). These probes labeled the corresponding three 
chromosome pairs of S. polyrhiza (Fig. 1b) and their homeologous counterparts of $S$. intermedia (clones 8410 and 7747), (Fig. 1c, d), confirming that $\mathrm{ChrSp} 08$ and $\mathrm{ChrSp} 18$ together correspond to $\mathrm{ChrSi09}$.

\section{Oligo-cross-FISH to other duckweed species}

After proving the chromosome specificity of oligo-probes in their species of origin and successful cross-FISH to homeologous Spirodela chromosomes, the same oligo-probe sets were applied to chromosome spreads of species of the other four duckweed genera. The studied species were (with increasing phylogenetic distance to the genus Spirodela): $L a$. punctata clone $7260(2 n=46 ; 424 \mathrm{Mbp} / 1 \mathrm{C})$, Le. aequinoctialis clone $2018(2 n=42 ; 452 \mathrm{Mbp} / 1 \mathrm{C}), W a$. hyalina clone $8640(2 n=40 ; 1234 \mathrm{Mbp} / 1 \mathrm{C})$, and $W o$. australiana clone $7540(2 n=40 ; 432 \mathrm{Mbp} / 1 \mathrm{C})$ (Hoang et al. 2019). No signals were detectable with the oligo-probe set specific for $\mathrm{ChrSi09beg}$ on either of the species, even not when structured illumination microscopy (SIM) was applied to achieve super-resolution. The probe specific for ChrSi09end and ChrSp19 generated dispersed signals over nearly the entire chromosome complements of La. punctata (Figs. 2 and S1), and Le. aequinoctialis. No signals were detectable on chromosomes of Wa. hyalina and Wo. australiana.

\section{Dispersed microsatellite sequences in Landoltia and Lemna target genomes apparently give rise to cross-FISH signals by the Spirodela-derived oligo-probes}

The dispersed signals of the $\mathrm{ChrSi} 09 \mathrm{end} / \mathrm{ChrSp} 19$ probe set on La. punctata and Le. aequinoctialis chromosomes suggest that some oligos are similar to dispersed repetitive sequences within the genomes of the two species. Indeed, some oligoprobe sequences contain for instance $(\mathrm{GA})_{\mathrm{n}}$ and $(\mathrm{GAA})_{\mathrm{n}}$

Table 1 Origin, number, and density of oligo-FISH probes expected to hybridize with homologous/homeologous regions of different duckweed genomes $^{\mathrm{a}}$

\begin{tabular}{|c|c|c|c|c|}
\hline Origin of probe set & Target species & Actual target region & Number of probes & median D100 (probes/Kb) \\
\hline \multirow[t]{6}{*}{ ChrSi09beg (green) } & S. polyrhiza $a^{b}(2 n=40,160 \mathrm{Mbp} / 1 \mathrm{C})$ & chrSp08:25541-8456612 & 27116 & 4.34 \\
\hline & S. intermedia ${ }^{c}(2 n=36,160 \mathrm{Mbp} / 1 \mathrm{C})$ & chrSi09:109671-77381134 & 27116 & 4.53 \\
\hline & La. punctata $^{d, e}(2 n=46,424 \mathrm{Mbp} / 1 \mathrm{C})$ & Whole genome & 2101 & \\
\hline & \multirow[t]{2}{*}{ Le. minor $^{f}(2 n=42,836 \mathrm{Mbp} / 1 \mathrm{C})$} & chrLem4B:126930-24098945 & 2052 & 0.10 \\
\hline & & chrLem4A:270877-17346499 & 1290 & 0.08 \\
\hline & Wo. $_{\text {australiana }}^{g}(2 n=40,432 \mathrm{Mbp} / 1 \mathrm{C})$ & chrWoa5:88567-22842065 & 1182 & 0.06 \\
\hline \multirow[t]{6}{*}{ ChrSi 09end (red) } & S. polyrhiza & chrSp18:159-5284411 & 13682 & 3.47 \\
\hline & S. intermedia & chrSi09:7793003-12619724 & 13682 & 3.69 \\
\hline & La. punctata & Whole genome & red: 1987 & \\
\hline & \multirow[t]{2}{*}{ Le. minor } & chrLem16A:67881-15943339 & 614 & 0.05 \\
\hline & & chrLem16B:62099-18968151 & 914 & 0.05 \\
\hline & Wo. australiana & chrWoa16:24408-13727754 & 543 & 0.05 \\
\hline \multirow[t]{7}{*}{ ChrSp19 (red) } & S. polyrhiza & chrSp19:30246-3907208 & 13696 & 5.01 \\
\hline & S. intermedia & chrSi17:43408-3929553 & 13696 & 5.24 \\
\hline & La. punctata & Whole genome & red: 1987 & \\
\hline & \multirow[t]{3}{*}{ Le. minor } & chrLem17B:6671746-15808965 & 282 & 0.08 \\
\hline & & chrLem20A:2922-9150510 & 636 & 0.07 \\
\hline & & chrLem20B:112225-7405979 & 693 & 0.10 \\
\hline & Wo. australiana & chrWoa4:4039-23215234 & 714 & 0.08 \\
\hline
\end{tabular}

\footnotetext{
${ }^{\text {a }}$ For inter-genus hybridization $>75 \%$ similarity is assumed

${ }^{\mathrm{b}}$ Hoang et al. (2018) for genomic data

${ }^{\mathrm{c}}$ Hoang et al. (2020) for genomic data of $S$. intermedia clones 7747 and 8410 see European Nucleotide Archive (ENA) under PRJEB35514 and PRJEB35634, respectively. Raw reads can be obtained from EBI ENA accession numbers PRJEB33624 (PacBio, S. intermedia 7747), ERR3829756 (Illumina, S. intermedia 8410), and ERR3957957-ERR3957958 (Oxford Nanopore, S. intermedia 8410).

${ }^{\mathrm{d}}$ For genomic data, see European Nucleotide Archive (https://www.ebi.ac.uk/ena) under accession number ERR4463159.

${ }^{\text {e}}$ For Landoltia, the probe values are probably overestimated due to "off-target" hits, because no chromosome assemblies are available.

${ }^{\mathrm{f}}$ Because no genomic data are available for Le. aequinoctialis, the genomic data for Le. minor lemna.org were used

${ }^{\mathrm{g}}$ Michael et al. (2020) bioRxiv https://doi.orh/10.1101/2020.03.31.018291 for genomic data
} 
microsatellite motifs (Table 2). A randomly sampled fraction of the Illumina reads was used to identify La. punctata repetitive elements employing RepeatExplorer and TAREAN pipelines (Novák et al. 2013; Novák et al. 2017). The analysis revealed a relatively small proportion of highly and moderately repeated sequences in La. punctata (38\% of the genome), with the prevalence of LTR-retrotransposons (20.9\%) and tandem repeats $(4.7 \%)$. The tandem repeats mainly consisted of microsatellite motifs $(\mathrm{GA})_{\mathrm{n}}$ and $(\mathrm{GAA})_{\mathrm{n}}$.

In order to check the chromosomal distribution of these microsatellite motifs on La. punctata, we performed FISH with labeled $(\mathrm{GA})_{15}$ and $(\mathrm{GAA})_{10}$ sequences as probes. Both probes hybridized to all chromosomes (Figs. 3 and S2) mostly in terminal regions as shown by the partial overlap with signals for the Arabidopsis-type telomere sequence repeat $\left(\mathrm{TTTAGG}_{\mathrm{n}}\right)$. One La. punctata satellite repeat revealed a monomer length of $138 \mathrm{bp}$ and an estimated abundance of $0.21 \%$ of the genome (LDP_SAT1). FISH with two partly overlapping oligos of $42 \mathrm{nt}$ (H1) and 47 nt (H2) of this GC-rich satellite sequence yielded similar signals as were obtained with labeled $(\mathrm{GA})_{15}$ and $(\text { GAA })_{10}$ probes (Fig. S3).

To reduce binding of oligos to dispersed repeats of $L a$. punctata, unlabeled (GA) 15 and (GAA) 10 sequences were added in excess (50 pmol of each microsatellite/20 pmol total labeled oligos of each chromosome/slide) to the probe. In spite of a reduction of dispersed signals, no specific labeling of distinct La. punctata chromosomes was recognizable (Fig. S4). Apparently, several further dispersed repeats of the target genomes match with Spirodela-derived oligos from ChrSi09end and/or ChrSp19 and yield dispersed signals on the chromosomes of La. punctata and Le. aequinoctialis.

\section{Computational probe mapping to other duckweed genera}

To predict the ability of the Spirodela-derived oligos to hybridize to chromosomes of other duckweed genera, probe sequences were blasted against the Le. minor (tetraploid) and Wo. australiana genomes. Le. minor is used here as a surrogate for Le. aequinoctialis, of which the genome is not yet sequenced. Best Blast hits with sequence similarity deemed enough to generate a stable hybridization (> 75\% similarity) were sorted by chromosomes (Table S1). Probes designed from ChrSi09beg preferentially match Le. minor chromosomes ChrLemA4 and B4 (1290 and 2055 hits, respectively), and Wo. australiana chromosome ChrWoa5 (1188 hits). Similarly, probes designed from ChrSi09end match Le. minor chromosomes ChrLemA16 and B16 (614 and 915 hits, respectively) and Wo. australiana chromosome ChrWoa16 (543 hits). Finally, probes designed from ChrSp19 match Le. minor chromosomes ChrLemB17, A20, and B20 (282, 636, and 693 hits, respectively) and Wo. australiana chromosome ChrWoa04 (715 hits). To acquire sequence information for evaluating similarities of Spirodela-based oligo-probes to their targets in the La. punctata genome, shotgun Illumina sequencing of the La. punctata genome (clone 7260) was performed and yielded 301.7 million pairs of raw Illumina reads $(2 \times 151 \mathrm{nt}$, ENA accession number ERR4463159), corresponding to $\mathrm{a} \sim 215$-fold genome coverage. The oligoprobe sequences were blasted against unassembled La.
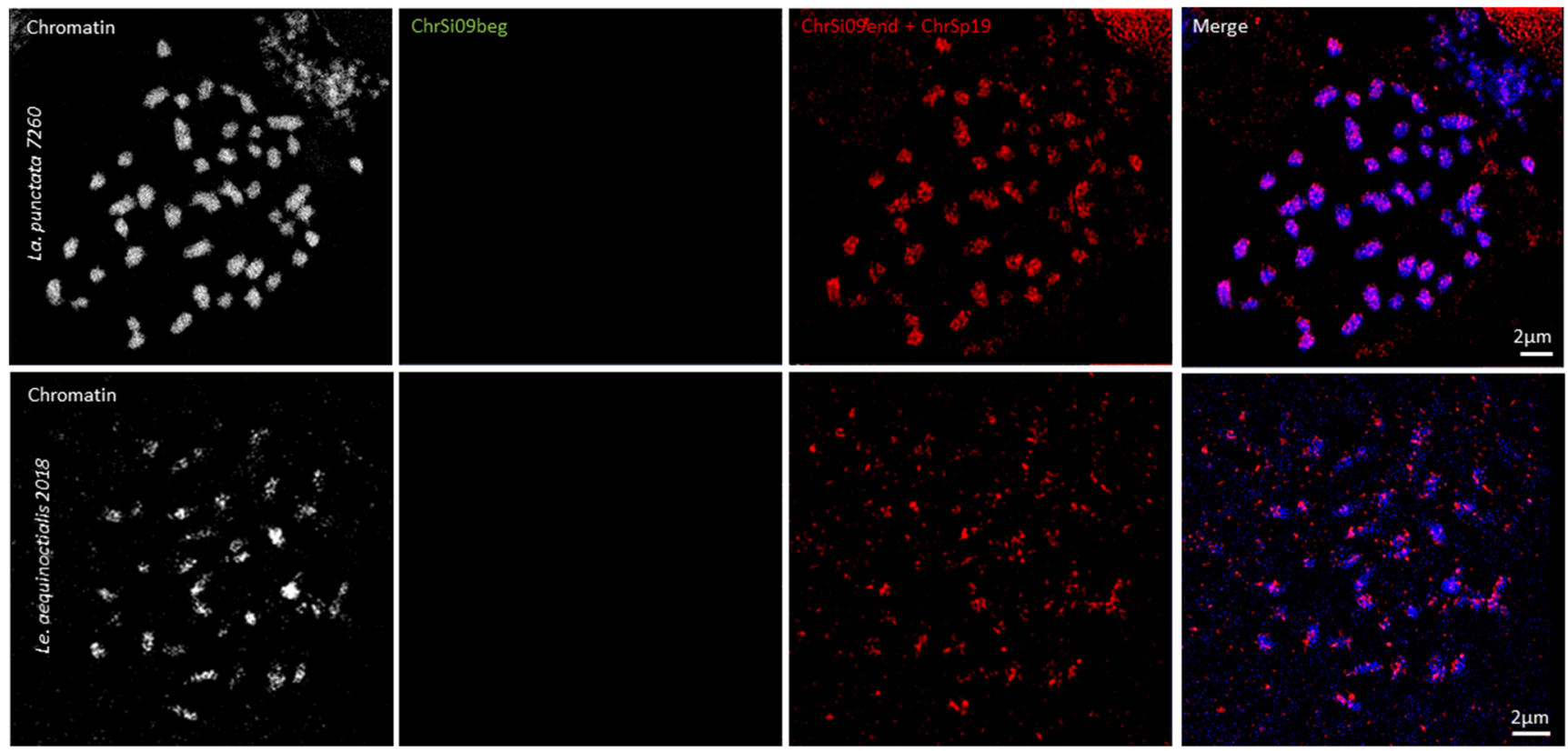

Fig. 2 Oligo-FISH with S. polyrhiza chromosome-specific probes on La. punctata (upper panel) and Le. aequinoctialis (lower panel). No green signals, but dispersed red signals were identified by spatial structured illumination microscopy (3D-SIM) 
Table 2 Examples of oligo-sequences containing microsatellite $(\mathbf{G A})_{\mathbf{n}}$ and (GAA) motifs

\begin{tabular}{|c|c|}
\hline ChrSp-specific probe & Oligo-sequences contain microsatellite motifs \\
\hline \multirow[t]{10}{*}{ ChrSp 08} & CCACGCTCAAGCAAGAACAAGACCCTTTGAGGAAGAAGAAGAAAC \\
\hline & AGAAGAAGAAGAAGCAATGGTATCCATGGAATTTCTTTCACCTAC \\
\hline & GGGGAAATTTCTGAGGATTTTTCTTGCTCTCCTTCTTCTTCTTCT \\
\hline & GAAGAAGAAGAAATAATGGTGCAGCTGGTTCGCTGTTCATCTTAG \\
\hline & GAAGAAGAAGAAAGCATTGGAAAGAAATCTTAGGAAGGTCGATGG \\
\hline & AGAGAGAGAGAGAAGTAGATAGGTGATGGCAAGATGGTCGTTGCG \\
\hline & TСТCTCTCTCTCTAAGATCCTCTTTGGTACATGGAAGGTACCGTG \\
\hline & AGAGAGAGAGAGAAATGCTCAAGACACATTTGACTTCTGCGTATG \\
\hline & GAGAGAGAGAGAGCATAGGGGTTCAATGTCTAGTGACTAGATGCC \\
\hline & GAGAGAGAGAGATTATCATGATGCTGTCTTATGTCAATCAAAGGC \\
\hline \multirow[t]{7}{*}{ ChrSp 18} & AGAAGAAGAAGAAGAGGATAGAACCGTTTGACGACCTCTCTTTCC \\
\hline & СтTCTTCTTCTTCTGGGTTCGATCAGTCTGTGCGTGAAGGGGTAC \\
\hline & AGGAGGAGCAGAAACCTAGAAGAAGAAGAAGCTCACGTTCTTCCG \\
\hline & CTTCTTCTTCTTCATAAGTCTACCGGCCGGATAACCAGAAGTGAG \\
\hline & AGAGAGAGAGAGAGTTAAATAGGACGTACAATTCCTACGAACCAA \\
\hline & СТСTCTCTCTCTACATTCTGGTGCATCGACACAGAGATAGATCCT \\
\hline & TCTCTCTCTCTCTTCGTTAAGAAAACATCTTGTTGGACTAGACGT \\
\hline \multirow[t]{7}{*}{ ChrSp 19} & AGTATCATCAAGTGAAGAAGAAGAATGCTTGACACAGGCTCGTTC \\
\hline & AAGAAGAAGAAGAAGCAATTGGAAAAAGAACTCGCGGCTGTCTGA \\
\hline & AAATCCAGCGATGGAACCATCATGGAGGTCCTTCTTCTTCTTCTT \\
\hline & GAGAGAGAGAGAGATGTAAATAAGTCAACTGGTGATGATGCCACT \\
\hline & СТСТCTCTCTCTAGAATACCATGCAGATCAGGAATGTGCAAAACC \\
\hline & AGAGAGAGAGAGAATTTGTGTGCAGTGACCGAGTCCTTACTCTCT \\
\hline & AGAGAAAGTACAGAGAGAGAGAGATTGAGGCACCTGAAGACCGGC \\
\hline
\end{tabular}

punctuata genome reads, generating 2010 and 1987 hits for ChrSi09beg and ChrSi09end/ChrSp19 probe sets, respectively. Due to the absence of an assembled genome for La. punctuata, likely the number of probes hybridizing
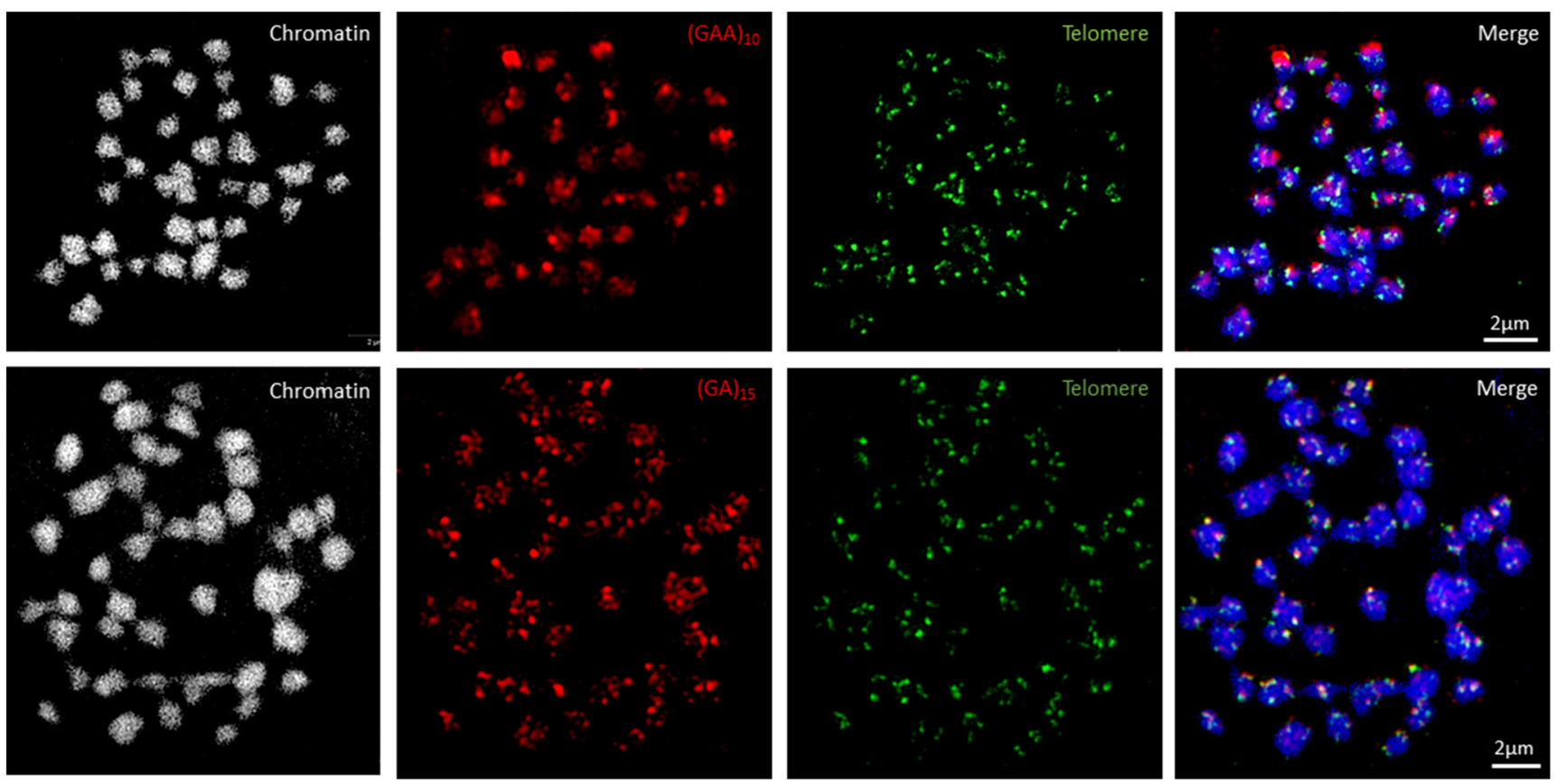

Fig. 3 Distribution of GAA and GA microsatellite signals on La. punctata chromosomes. GAA (upper panel), GA (lower panel) microsatellite probes, and telomere repeats (TTTAGGG); imaged by 3D-SIM 
to homeologous chromosomes is overestimated since 5 to $15 \%$ of all hits on Le. minor and Wo. australiana genomes do not land on preferential chromosomes (Table S1). The small number of probes able to hybridize to Le. minor and Wo. australiana genomes and the fact they are spread across multiple chromosomes lead to very low median D100 probe densities ( 0.05 to 0.1 probes $/ \mathrm{kb}$, see Table 1, Fig. S6) compared to hybridizations between the Spirodela species. Most likely the same is true for La. punctuata.

\section{Discussion}

Our oligo-cross-FISH experiments yielded strong and specific signals only within the genus Spirodela. The absence of chromosome-specific signals after cross-FISH across the genus border is likely due to the low number of probes with enough sequence similarity to achieve a stable hybridization between the probe and the chromosomes of the tested species. Similarly, while probes of $>90 \%$ similarity equally well labeled homeologous chromosomes of the allotetraploid switchgrass Panicum virgatum, probes of $<75 \%$ similarity yielded virtually no signals on homeologs (Jiang 2019).
An alternative or additional explanation might be a too large distance between probes hybridizing to the target chromosome to generate a detectable signal. We computationally demonstrated that the number of probes able to stably hybridize to chromosomes across the genus border is strongly reduced compared to intra-genus hybridization. This is leading to a 40 - to 80 -fold reduction in probe density along the target sequence. It is likely that the reduced number of probes able to hybridize to homeologous chromosome regions of species across the genus border is too distantly located along the target chromosomes to generate detectable chromosome-specific signals. Although successful oligo-FISH with various densities (0.1-0.5 oligos/kb; Jiang 2019) has been reported for different plant species, and Song et al. (2020) found even 0.052 oligos $/ 1 \mathrm{~kb}$ of the target chromosome $4 \mathrm{D}$ of wheat sufficient for reliable chromosome-specific labeling, such low density of oligo-sequences did not generate reliable FISH signals in cross-hybridization between duckweed genera which apparently have a less dense chromosome structure than wheat. Similarly, Simonikova et al. (2019) found in banana chromosome complements that a density of 0.8 oligos $/ \mathrm{kb}$ did no longer label target chromosome regions contiguously. Albeit also technical details of oligo-FISH approaches could influence the

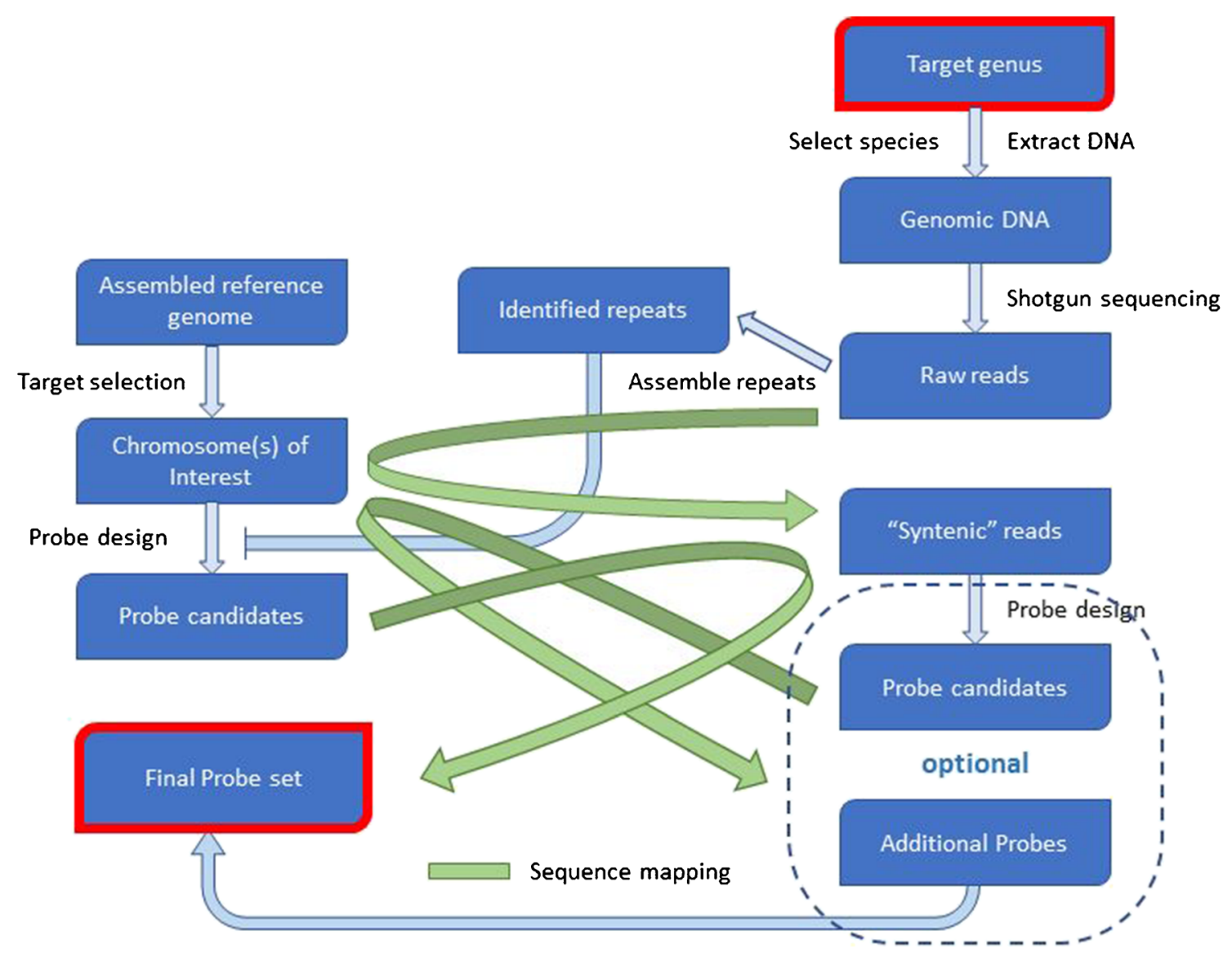

Fig. 4 Proposed workflow for designing probes for oligo-FISH across genera 
results, sequence similarity and probe density have to be optimally adjusted for each case of oligo-cross painting.

Because a bioinformatic comparison of the different probe sets did not reveal any differences that could explain why only the $\mathrm{ChrSi09end/ChrSp19}$ probe set leads to such dispersed signal and not the ChrSi09beg probe set, possibly, the ATTO-488-labeled probe set signal was weaker than the one from the ATTO-594-labeled set and therefore yielded no detectable signals across the genus border.

Probes used in the present study were designed using only Spirodela species genomes to predict and exclude sequences capable of forming non-specific hybridizations. Our observation of dispersed signals in Landoltia and Lemna and lack of specific chromosome labeling across the genus border support the need to also include genomic sequences from species of other genera and chromosome synteny data during the probe selection process. Unassembled reads from a relatively inexpensive shallowdepth shotgun sequencing should provide enough information to select probes that can produce strong and specific signals in multiple genera, granted that at least one species included in the study has been sequenced and fully assembled.

We propose the following workflow to design FISH probes for studies across genera (Fig. 4). The genomic DNA from a representative species for each target genus should be shotgun-sequenced. A shallow 5-10 $\times$ coverage should provide enough data to identify the most common repeats present in that genome. The reads should also be mapped to the reference species genome. Reads mapping preferentially to the chromosome(s) of interest should be selected as reads from syntenic regions. Probes should be designed against the chromosomes of interest from the reference species. Candidate probes should be checked for lack of cross-hybridization against the repeat sequences obtained from the newly sequenced species. Candidate probes should be also mapped to the syntenic reads to select probes with greater than $85 \%$ homology with the other genus sequence. If more probes are needed, an optional probe design could be done using the syntenic reads as input. These additional candidate probes should be compared to the reference genome to ensure they are specific to the intended reference target chromosome and can hybridize to species from both genera. More elaborated design workflows could involve assembling overlapping reads into larger contigs to expand the probe design space in the newly assembled genome. This may be helpful in designing probes for phylogenetically distant genera.

\section{Conclusions}

Oligo-probes (as well as BACs) yielded chromosome-specific FISH signals within duckweed species of the same genus, but not across genus borders, apparently because of too low density of oligos sufficiently similar to the target chromosome sequences. Minisatellite motifs within the probes may yield dispersed FISH signals, when abundant in the target genome. Oligos containing such motifs should be filtered out. If no assembled genomes are available for the genus of the target species and oligo-FISH across the genus border does not give chromosome-specific results, oligo-probes should be designed from shotgun sequences based on synteny with a related genus. Suitability of probes should be validated by FISH on homologous chromosomes before applied for congeneric karyotyping and identification of homeologous/rearranged chromosomes of congeneric species.

Supplementary Information The online version contains supplementary material available at https://doi.org/10.1007/s00412-020-00749-2.

Acknowledgments We thank Todd Michael (JCVI, San Diego, USA) for his help with translation of synteny data into the actual chromosome nomenclature of Lemna minor and Wolffia australiana, and Andrea Kobližǩvá (Biology Centre, České Budějovice, Czech Republic) for isolating genomic DNA from L. punctata and one anonymous reviewer for very helpful suggestions.

Author contributions PNTH and IS designed experiments; PNTH and IK performed cytogenetic experiments, PNTH, IS, JMR, JM, IK, and VS analyzed data; PNTH and IS wrote the manuscript with contribution of all authors. All authors read and approved the manuscript.

Funding Open Access funding enabled and organized by Projekt DEAL. This work was supported by a grant of the German Research Foundation [SCHU 951/18-1] to IS. PTNH was supported by the Vietnam National Foundation for Science and Technology Development (NAFOSTED) grant \# 106.01-2020.33.

\section{Compliance with ethical standards}

Conflict of interest PNTH, JM, IK, VS, and IS declare to have no conflict of interest. JMR is an employee of Arbor Biosciences. Arbor Biosciences designed and manufactured probe sets for this study.

Open Access This article is licensed under a Creative Commons Attribution 4.0 International License, which permits use, sharing, adaptation, distribution and reproduction in any medium or format, as long as you give appropriate credit to the original author(s) and the source, provide a link to the Creative Commons licence, and indicate if changes were made. The images or other third party material in this article are included in the article's Creative Commons licence, unless indicated otherwise in a credit line to the material. If material is not included in the article's Creative Commons licence and your intended use is not permitted by statutory regulation or exceeds the permitted use, you will need to obtain permission directly from the copyright holder. To view a copy of this licence, visit http://creativecommons.org/licenses/by/4.0/.

\section{References}

Appenroth KJ, Sree KS, Bohm V, Hammann S, Vetter W, Leiterer M, Jahreis G (2017) Nutritional value of duckweeds (Lemnaceae) as human food. Food Chem 217:266-273. https://doi.org/10.1016/j. foodchem.2016.08.116 
Appenroth K-J, Teller S, Horn M (1996) Photophysiology of turion formation and germination inSpirodela polyrhiza. Biol Plant 38:95106. https://doi.org/10.1007/bf02879642

Aurich-Costa J, Zamechek L, Keenan P, Bradley S (2007) Oligo fluorescence in situ hybridization (oligo-fish), a new strategy for enumerating chromosomes in interphase nuclei. Fertil Steril 88:S86. https:// doi.org/10.1016/j.fertnstert.2007.07.287

Bog M, Sree KS, Fuchs J, Hoang PTN, Schubert I, Kuever J, Rabenstein A, Paolacci S, Jansen MAK, Appenroth KJ (2020) A taxonomic revision of Lemna sect. Uninerves (Lemnaceae). Taxon 69:56-66. https://doi.org/10.1002/tax.12188

Braz GT et al (2018) Comparative oligo-FISH mapping: an efficient and powerful methodology to reveal karyotypic and chromosomal evolution. Genetics 208:513-523. https://doi.org/10.1534/genetics.117. 300344

Cao HX, Vu GT, Wang W, Appenroth KJ, Messing J, Schubert I (2016) The map-based genome sequence of Spirodela polyrhiza aligned with its chromosomes, a reference for karyotype evolution. New Phytol 209:354-363. https://doi.org/10.1111/nph.13592

de Beukelaar MFA, Zeinstra GG, Mes JJ, Fischer ARH (2019) Duckweed as human food. The influence of meal context and information on duckweed acceptability of Dutch consumers. Food Qual Prefer 71:76-86. https://doi.org/10.1016/j.foodqual.2018.06.005

Han Y, Zhang T, Thammapichai P, Weng Y, Jiang J (2015) Chromosome-specific painting in Cucumis species using bulked oligonucleotides. Genetics 200:771-779. https://doi.org/10.1534/ genetics.115.177642

Harkess A, McLaughlin F, Bilkey N, Elliot K, Emenecker R, Mattoon E, Miller K, Czymme KK, Vierstra R, Meyers BC, Michael TP (2020) A new Spirodela polyrhiza genome and proteome reveal a conserved chromosomal structure with high abundances of proteins favoring energy production. bioRxiv. https://doi.org/10.1101/2020. 01.23.909457

Hoang PTN (2019) Comparative cytology and cytogenomics for representative species of the five duckweed genera., Halle/S., MartinLuther-Universität Halle-Wittenberg

Hoang PTN, Fiebig A, Novák P, Macas J, Cao HX, Stepanenko A, Chen G, Borisjuk N, Scholz U, Schubert I (2020) Chromosome-scale genome assembly for the duckweed Spirodela intermedia, integrating cytogenetic maps, PacBio and Oxford Nanopore libraries. Sci Rep 10:19230. https://doi.org/10.1038/s41598-020-75728-9

Hoang PTN, Schubert I (2017) Reconstruction of chromosome rearrangements between the two most ancestral duckweed species Spirodela polyrhiza and S. intermedia. Chromosoma 126:729-739. https://doi. org/10.1007/s00412-017-0636-7

Hoang PTN, Schubert V, Meister A, Fuchs J, Schubert I (2019) Variation in genome size, cell and nucleus volume, chromosome number and rDNA loci among duckweeds. Sci Rep 9:3234. https://doi.org/10. 1038/s41598-019-39332-w

Hoang PNT et al (2018) Generating a high-confidence reference genome map of the Greater Duckweed by integration of cytogenomic, optical mapping and Oxford Nanopore technologies. Plant J 96:670 684. https://doi.org/10.1111/tpj.14049

Ijdo JW, Wells RA, Baldini A, Reeders ST (1991) Improved telomere detection using a telomere repeat probe (TTAGGG)n generated by PCR. Nucleic Acids Res 19:4780

Jiang JM (2019) Fluorescent in situ hybridization in plants: recent developments and future directions. Chromosome Res 27:153-165. https://doi.org/10.1007/s10577-019-09607-z

Kaur M, Srikanth S, Kumar M, Sachdeva S, Puri SK (2019) An integrated approach for efficient conversion of Lemna minor to biogas. Energy Convers Manag 180:25-35. https://doi.org/10.1016/j.enconman. 2018.10.106
Kreider AN, Fernandez Pulido CR, Bruns MA, Brennan RA (2019) Duckweed as an agricultural amendment: nitrogen mineralization, leaching, and sorghum uptake. J Environ Qual 48:469-475. https:// doi.org/10.2134/jeq2018.05.0207

Landolt E (1986) The family of Lemnaceae - a monographic study (Vol 1) Veröffentlichungen des Geobotanischen Institutes der Eidg Techn Hochschule, Zürich

Liu X et al (2020) Dual-color oligo-FISH can reveal chromosomal variations and evolution in Oryza species. Plant J: Cell Mol Biol 101: 112-121. https://doi.org/10.1111/tpj.14522

Lysak MA, Berr A, Pecinka A, Schmidt R, McBreen K, Schubert I (2006) Mechanisms of chromosome number reduction in Arabidopsis thaliana and related Brassicaceae species. Proc Natl Acad Sci U S A 103:5224-5229. https://doi.org/10.1073/pnas.0510791103

Ma L, Vu GT, Schubert V, Watanabe K, Stein N, Houben A, Schubert I (2010) Synteny between Brachypodium distachyon and Hordeum vulgare as revealed by FISH. Chromosome Res: Int J Mol, Supramol Evol Asp Chromosome Biol 18:841-850. https://doi. org/10.1007/s10577-010-9166-3

Mandakova T, Lysak MA (2008) Chromosomal phylogeny and karyotype evolution in $\mathrm{x}=7$ crucifer species (Brassicaceae). Plant Cell 20: 2559-2570. https://doi.org/10.1105/tpc.108.062166

Michael TP et al (2017) Comprehensive definition of genome features in Spirodela polyrhiza by high-depth physical mapping and short-read DNA sequencing strategies. Plant J: Cell Mol Biol. https://doi.org/ 10.1111/tpj.13400

Michael TP, Ernst E, Hartwick N, Chu P, Bryant D, Gilbert S, Ortleb S, Baggs EL, Sree KS, Appenroth KJ, Fuchs J, Jupe F, Sandoval JP, Krasileva KV, Borisjuk L, Mockler TC, Ecker JR, Martiessen RA, Lam E (2020) Genome and time-of-day transcriptome of Wolffia australianalink morphologial extreme limitization with un-gated plant growth. bioRxiv 2020.03.31.018291. https://doi.org/10.1101/ 2020.03.31.018291

Nahar K, Sunny SA (2020) Duckweed-based clean energy production dynamics (ethanol and biogas) and phyto-remediation potential in Bangladesh. Model Earth Syst Environ 6:1-11. https://doi.org/10. 1007/s40808-019-00659-y

Novák P, Ávila Robledillo L, Koblížková A, Vrbová I, Neumann P, Macas J (2017) TAREAN: a computational tool for identification and characterization of satellite DNA from unassembled short reads. Nucleic Acids Res 45:e111. https://doi. org/10.1093/nar/gkx257

Novák P, Neumann P, Pech J, Steinhaisl J, Macas J (2013) RepeatExplorer: a galaxy-based web server for genome-wide characterization of eukaryotic repetitive elements from next-generation sequence reads. Bioinformatics 29:792-793. https://doi.org/10. 1093/bioinformatics/btt054

Simonikova D, Nemeckova A, Karafiatova M, Uwimana B, Swennen R, Dolezel J, Hribova E (2019) Chromosome painting facilitates anchoring reference genome sequence to chromosomes in situ and integrated karyotyping in banana (Musa Spp.). Front Plant Sci 10: 1503. https://doi.org/10.3389/fpls.2019.01503

Song X, Song R, Zhou J, Yan W, Zhang T, Sun H, Xiao J, Wu Y, Xi M, Lou Q, Wang H, Wang X (2020) Development and application of oligonucleotide-based chromosome painting for chromosome 4D of Triticum aestivum L. Chromosom Res 28:171-182. https://doi.org/ 10.1007/s10577-020-09627-0

Tippery NP, Les DH, Crawford DJ (2015) Evaluation of phylogenetic relationships in Lemnaceae using nuclear ribosomal data. Plant Biol 17(Suppl 1):50-58. https://doi.org/10.1111/plb.12203

Wang W, Haberer G, Gundlach H, Gläßer C, Nussbaumer T, Luo MC, Lomsadze A, Borodovsky M, Kerstetter RA, Shanklin J, Byrant DW, Mockler TC, Appenroth KJ, Grimwood J, Jenkins J, Chow J, 
Choi C, Adam C, Cao XH, Fuchs J, Schubert I, Rokhsar D, Schmutz J, Michael TP, Mayer KFX, Messing J (2014) The Spirodela polyrhiza genome reveals insights into its neotenous reduction fast growth and aquatic lifestyle. Nat Commun 5:3311. https://doi.org/ $10.1038 /$ ncomms 4311

Wang W, Kerstetter RA, Michael TP (2011) Evolution of genome size in Duckweeds (Lemnaceae). J Bot 2011:1-9. https://doi.org/10.1155/ 2011/570319

Weisshart K, Fuchs J, Schubert V (2016) Structured illumination microscopy (SIM) and photoactivated localization microscopy (PALM) to analyze the abundance and distribution of RNA polymerase II molecules on flow-sorted arabidopsis Nuclei. Bio-protocol 6:e1725. https://doi.org/10.21769/BioProtoc.1725

Xin H, Zhang T, Wu Y, Zhang W, Zhang P, Xi M, Jiang J (2020) An extraordinarily stable karyotype of the woody Populus species revealed by chromosome painting. Plant J : Cell Mol Biol 101: 253-264. https://doi.org/10.1111/tpj.14536

Yaskolka Meir A et al (2019) A green-mediterranean diet, supplemented with Mankai Duckweed, preserves iron-homeostasis in humans and is efficient in reversal of anemia in Rats. J Nutr 149:1004-1011. https://doi.org/10.1093/jn/nxy321

Zhao Y et al (2015) Pilot-scale comparison of four duckweed strains from different genera for potential application in nutrient recovery from wastewater and valuable biomass production. Plant Biol 17(Suppl 1):82-90. https://doi.org/10.1111/plb.12204

Publisher's note Springer Nature remains neutral with regard to jurisdictional claims in published maps and institutional affiliations. 